\title{
Worst-case tolerance optimization of antenna systems
}

\section{Schjær-Jacobsen, Hans}

\section{Published in:}

I E E E Transactions on Antennas and Propagation

Link to article, DOI:

10.1109/TAP.1980.1142296

Publication date:

1980

Document Version

Publisher's PDF, also known as Version of record

Link back to DTU Orbit

Citation (APA):

Schjær-Jacobsen, H. (1980). Worst-case tolerance optimization of antenna systems. I E E E Transactions on Antennas and Propagation, 28(2), 247-250. https://doi.org/10.1109/TAP.1980.1142296

\section{General rights}

Copyright and moral rights for the publications made accessible in the public portal are retained by the authors and/or other copyright owners and it is a condition of accessing publications that users recognise and abide by the legal requirements associated with these rights.

- Users may download and print one copy of any publication from the public portal for the purpose of private study or research.

- You may not further distribute the material or use it for any profit-making activity or commercial gain

- You may freely distribute the URL identifying the publication in the public portal

If you believe that this document breaches copyright please contact us providing details, and we will remove access to the work immediately and investigate your claim 


\section{Worst-Case Tolerançe Optimization of Antenna Systems}

\section{HANS SCHJAER-JACOBSEN, MEMBER, IEEE}

\begin{abstract}
The application of recently developed algorithms to antenna systems design is demonstrated by the worst-case tolerance optimization of linear broadside arrays, using both spacings and excitation coefficients as design parameters. The resulting arrays are optimally immunized against deviations of the design parameters from their nominal values.
\end{abstract}

\section{INTRODUCTION}

In this communication we shall be concerned with the optimization of antenna systems where the optimization parameters are subject to tolerances. To the author's knowledge no such attempts have been made within the antenna area before. In the electical circuit design area, however, a number of papers may be found dealing with tolerance optimization and related problems, a few of them being listed, [1] [7]. Recently, algorithms developed by Madsen and Schjaer-Jacobsen were described [8] and the programs were documented [9]. In the present work these algorithms are applied to antenna systems design.

The classical minimax antenna synthesis problem, which in the present context shall be denoted as the zero tolerance problem (ZTP) may be formulated as follows [10]. The desired antenna pattern is a function of the field coordinates $\psi$

$$
P_{D}=P_{D}(\psi), \quad \psi=\left(\psi_{1}, \psi_{2}, \psi_{3}\right)^{T}
$$

The $n$ optimization parameters are elements in the real vector $\mathbf{x}$ and the calculated pattern is denoted

$$
P=P(\mathrm{x}, \psi), \quad \mathrm{x}=\left(x_{1}, \cdots, x_{n}\right)^{T}
$$

Given a set of $m$ field coordinates $\psi_{1}, \cdots, \psi_{m}$, a set of $m$ nonlinear functions is defined by

$$
f_{j}(\mathbf{x})=w_{j}\left(P\left(\mathbf{x}, \psi_{j}\right)-P_{D}\left(\psi_{j}\right)\right), \quad j=1, \cdots, m,
$$

where $w_{j}$ are weights. The ZTP then consists of minimizing with respect to $\mathrm{x}$ the objective function

$$
F(\mathbf{x})=\max _{1 \leqslant j \leqslant m}\left|f_{j}(\mathbf{x})\right|
$$

A solution to the ZTP shall be denoted $x^{*}$, such that

$$
F^{*}=F\left(\mathbf{x}^{*}\right)=\min _{\mathbf{x} \in R^{n}} F(\mathbf{x})
$$

Manuscript received June 15, 1978; revised July 3, 1979. This work was supported by the Danish Natural Science Research Council under Grant 511-6874.

The author was with the Institute for Numerical Analysis, Technical University of Denmark, Lyngby, Denmark. He is now with A/S LK-NES, Industriparken 20-36, DK-2750 Ballerup, Denmark.

\section{PROBLEM FORMULATION AND METHOD OF SOLUTION}

For easy notation let us introduce the integer index sets

$$
I=\{i \mid i=1, \cdots, n\}, \quad J=\{j \mid j=1, \cdots, m\}
$$

Let there be given a vector of tolerances

$$
\delta=\left(\delta_{1}, \cdots, \delta_{n}\right)^{T}, \quad \delta_{i} \geqslant 0, \quad \text { for } i \in I,
$$

on the parameter vector $\mathbf{x}$. The tolerance interval is defined as

$$
\Omega_{\mathbf{x}, \eta}=\left\{\mathbf{y} \mid\left(x_{i}-\eta \delta_{i} \leqslant y_{i} \leqslant x_{i}+\eta \delta_{i}, \eta \geqslant 0, i \in I\right)\right\},
$$

and the worst-case objective function is defined as

$$
F_{\eta}(\mathbf{x})=\max _{j \in J, \mathbf{y} \in \Omega \mathbf{x}, \eta} f_{j}(\mathbf{y}), \quad \mathrm{y}=\left(y_{1}, \cdots, y_{n}\right)^{T}
$$

Notice that $x$ is now the "center" of the tolerance interval ( $x$ contains the nominal values of the design parameters) and that $\eta$ may be interpreted as a scaling factor of the tolerance interval. For given values of $\mathrm{x}$ and $\eta$ the problem of determining the worst-case objective function (9) shall be identified as the worst-case problem (WCP).

The function $f_{j}(\mathrm{x})$ is a measure of the discrepancy between the desired and the actual pattern at the $j^{\text {th }}$ sample point in space for a set of nominal design parameters $\mathbf{x}$. Therefore $F_{\eta}(\mathrm{x})$ may be interpreted as the largest discrepancy among all $m$ samples when the parameters vary within the tolerance interval.

The fixed tolerance problem (FTP) is now defined as that of minimizing the worst-case objective function (9) with respect to $\mathbf{x}$, i.e., determining $\mathrm{x}_{\eta}{ }^{*}$ such that

$$
F_{\eta}^{*}=F_{\eta}\left(\mathbf{x}_{\eta}^{*}\right)=\min _{\mathbf{x} \in R^{n}} F_{\eta}(\mathbf{x}), \quad \eta \text { fixed. }
$$

In other words, the nominal values of the design parameters are determined in such a way that the maximum of the functions $f_{j}$ calculated within the tolerance interval is minimized.

The solution of the WCP involves a global optimization problem within the tolerance interval. In the algorithms [8] this problem is solved either by imposing simplifying assumptions on the functions $f_{i}$ or by using interval arithmetic. The FTP is of the same structure as the ZTP and is solved by an algorithm which is similar to the one described in [11]. For further details the reader is referred to [8].

The FTP was defined with a fixed size of the tolerance interval. We now intend to let the size of the tolerance interval vary, in fact, to make it as large as possible. The variable tolerance problem (VTP) is defined as that of determining

$$
\eta^{*}=\max \eta
$$

subject to the constraint

$$
F_{\eta} * \leqslant c,
$$

where the given constant $c$ has to be larger than $F^{*}$ for obvious 
reasons. Essentially, we have defined the VTP as a one-dimensional problem of maximizing a single parameter, namely $\eta$, subject to a nonlinear constraint which is expressed in terms of the solution to the corresponding FTP.

The algorithm proposed in [8] for the VTP is based on repeated application of the previously described algorithm for the FTP. The problem is to determine the intersection between the function $F_{\eta}{ }^{*}$ and the constant $c$ (see (11) and (12)). This is done by a Regula-Falsi method where each iteration involves one solution of the FTP. We thus simultaneously determine the maximum value of $\eta$, called $\eta^{*}$, and the center of the tolerance interval $\mathrm{x}=\mathrm{x}^{*}{ }_{\eta=\eta} \eta_{1}$ under the condition that the constraint (12) is satisfied.

The algorithms have been implemented in double precision Fortran IV on an IBM $370 / 165$ computer carrying about 16 decimal digits [9]. The user must program the functions $f_{j}$ and their partial derivatives and submit an initial value $x_{0}$ of the vector $\mathbf{X}$, an initial step length $\Lambda_{0}$ for the FTP algorithm, and a stopping criterion $\epsilon$. In the case of a VTP the constant $c$ also has to be specified. The parameter $\eta$ is initially given the value of unity.

\section{OPTIMIZATION OF LINEAR BROADSIDE ARRAYS}

\section{The Normalized Radiation Pattern}

Consider symmetrical arrays with $N$ elements (Fig. 1). The normalized radiation pattern may be written

$$
f_{j}=\frac{A}{B}
$$

where

$$
\begin{aligned}
& A=a_{0}+2 \sum_{i=1}^{i_{N}} a_{i} \cos \left(2 \pi x_{i} \sin \theta_{j}\right), \\
& B=a_{0}+2 \sum_{i=1}^{i_{N}} a_{i}, \\
& a_{0}= \begin{cases}0, & N \text { even }, \\
1, & N \text { odd },\end{cases} \\
& i_{N}=\left\{\begin{array}{cc}
\frac{N}{2}, & N \text { even }, \\
\frac{N-1}{2}, & N \text { odd } .
\end{array}\right.
\end{aligned}
$$

Both the real excitation coefficients $a_{i}$ and the elements positions $x_{i}$ may be parameters. Therefore the partial derivatives

$$
\frac{\partial f_{j}}{\partial x_{i}}=\left(-4 \pi a_{i} \sin \theta_{j} \sin \left(2 \pi x_{i} \sin \theta_{j}\right)\right) / B
$$

and/or

$$
\frac{\partial f_{j}}{\partial a_{i}}=2\left(B \cos \left(2 \pi x_{i} \sin \theta_{j}\right)-A\right) / B^{2}
$$

are needed.
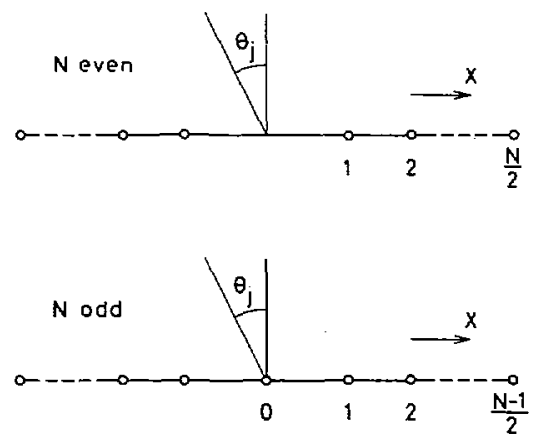

Fig. 1. Symmetrical broadside arrays with $N$ elements.

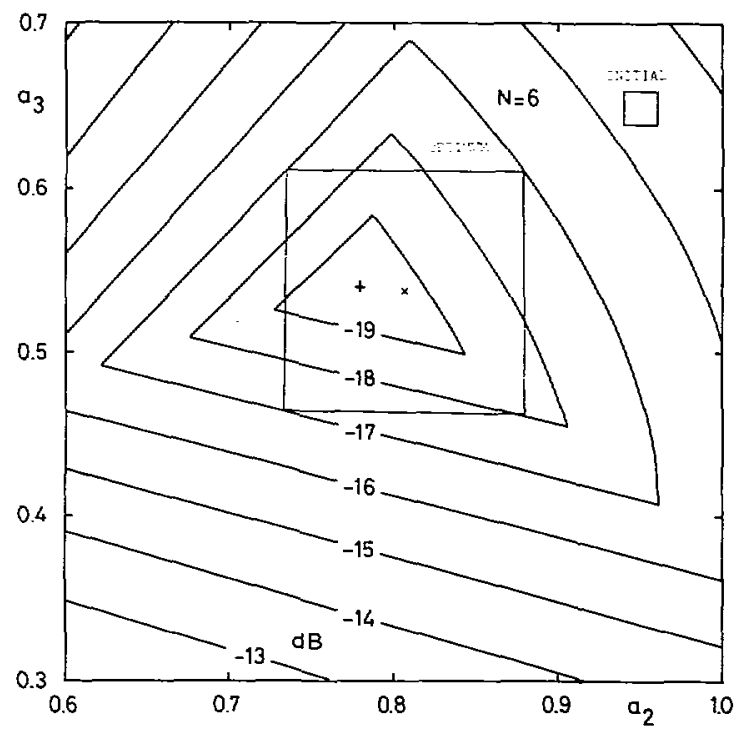

Fig. 2. Contour plot of zero tolerance objective function for sixelement array. Initial and optimum tolerance intervals.

\section{Uniformly Spaced Arrays}

In this section the element position vector is constant and we consider $(\lambda / 2)$-spaced arrays [12]. For such arrays the Dolph-Chebyshev excitation coefficients may be calculated using the formula given by Stegen [13].

For example, if $N=6$ and the desired sidelobe is $-20 \mathrm{~dB}$, we get for the normalized excitation $\left(a_{1}, a_{2}, a_{3}\right)^{T}=(1.0$, $0.7768,0.5406)^{T}$. Our purpose is to vary the excitation coefficients in order to optimize the array in the worst-case sense. The zero tolerance function

$$
F\left(a_{2}, a_{3}\right)=\max _{j \in J}\left|f_{j}\left(a_{2}, a_{3}\right)\right|, \quad a_{1}=1,
$$

is shown in Fig. 2, where the angles $\theta_{j}$ are essentially chosen with $0.5^{\circ}$ spacing, but the angles where the pattern attains its maxima are also included [10]:

$$
\begin{array}{r}
\left(\theta_{1}, \cdots, \theta_{m}\right)^{T}=(21.11,21.5,22.0, \cdots 31.43, \cdots, \\
56.30, \cdots, 90.0)^{T}, \quad m=141 .
\end{array}
$$

Let there be given the VTP; determine the excitation coefficients $a_{2}$ and $a_{3}$ and maximize their tolerances such that the worst-case sidelobe level is $-17 \mathrm{~dB} \sim c=0.14125$. As initial conditions we choose $a_{2}=0.95, a_{3}=0.65, \delta=(0.01$, $0.01)^{T}, \eta=1, \Lambda_{0}=0.05$, and $\epsilon=10^{-4}$. After 15 iterations 


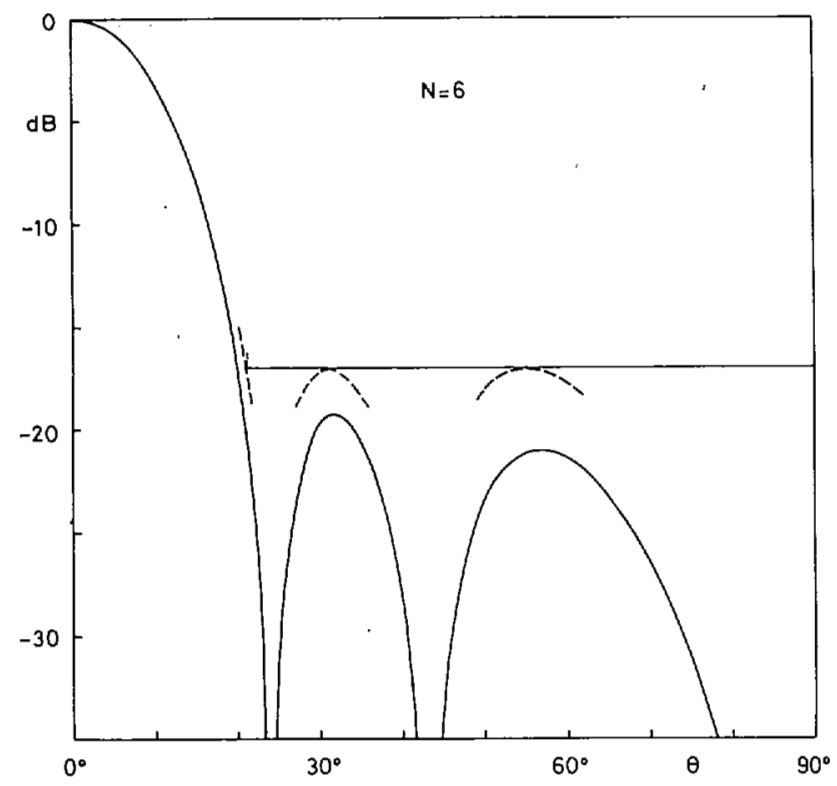

Fig. 3. Radiation pattern for optimized six-element array calculated at nominal values $\left(a_{1}, a_{2}, a_{3}\right)=(1,0.8066,0.5368)$. Worst-case pattern indicated by dotted curves.

we get the solution $a_{2}{ }^{*}=0.8066, a_{3} *=0.5368$, and $\eta^{*}=$ 7.300. The initial point and the solution together with their corresponding tolerance intervals are depicted in Fig. 2. In Fig. 3 the radiation pattern calculated at the nominal value of the solution is shown as well as the critical part of the worst-case pattern for the excitation $(1.0,0.8066 \pm 0.073,0.5368 \pm$ 0.073 ). It is seen from either figure that inside the optimum tolerance interval the sidelobe level is below $-17 \mathrm{~dB}$, as it should be.

Next we consider a 14-element array and the zero tolerance function

$$
F\left(a_{2}, \cdots, a_{7}\right)=\max _{j \in J}\left|f_{j}\left(a_{2}, \cdots, a_{7}\right)\right|, \quad a_{1}=1,
$$

where the sample angles correspond to a $-20-\mathrm{dB}$ sidelobe level:

$$
\begin{aligned}
\left(\theta_{1}, \cdots, \theta_{m}\right)^{T}= & (8.355,8.5,9.0, \cdots, 12.16, \cdots, 19.74, \cdots, \\
& 28.68, \cdots, 38.81, \cdots, 50.85, \cdots, 67.68, \cdots, \\
& 90.0)^{T}, \quad m=171 .
\end{aligned}
$$

Again we solve a VTP with $c=0.14125$, and the initial conditions are $a_{2}=\cdots=a_{7}=1.0, \delta_{i}=0.01, \eta=1, \Lambda_{0}=0.05$, and $\epsilon=10^{-4}$. After 14 iterations the solution $\left(a_{2}, * \cdots, a_{7}\right)^{T}=$ $(0.9632,0.8859,0.7973,0.6651,0.5549,0.7675)^{T}$ and $\eta^{*}=6.428$ is found. The corresponding radiation pattern is shown in Fig. 4 together with the critical parts of the worstcase pattern, the maxima of which are $-17.00 \mathrm{~dB}$. The excitation coefficients found may be compared with those corresponding to the zero tolerance $-20-\mathrm{dB}$ Chebyshev pattern [13].

\section{Nonuniformly Spaced Arrays}

In this section the array excitations are kept constant,

$$
a_{i}=1 \text {, }
$$

and the element positions $x_{i}$ are varied.

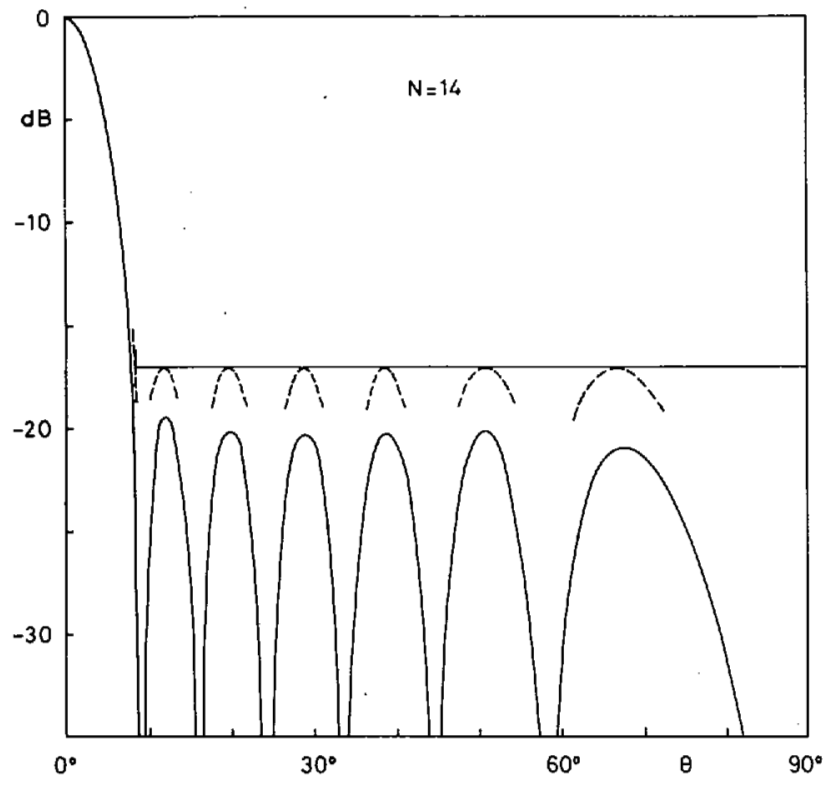

Fig. 4. Radiation pattern for optimized 14-element array calculated at nominal values $\left(a_{1}, \cdots, a_{7}\right)=(1,0.9632,0.8859,0.7973,0.6651$, $0.5549,0.7675)$. Worst-case pattern indicated by dotted curves.

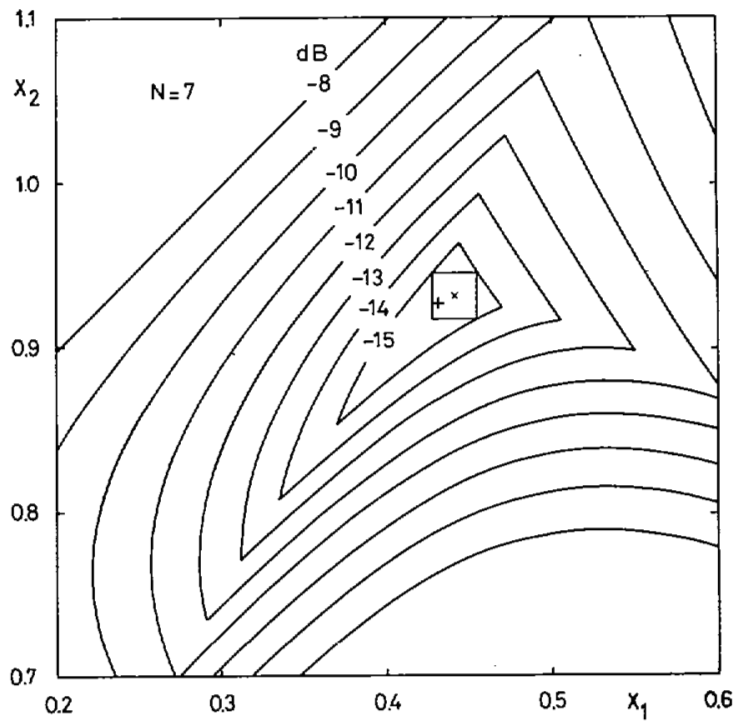

Fig. 5. Contour plot of zero tolerance objective function for sevenelement array.

First, $N=7$, and we consider the zero tolerance objective function

$$
F\left(x_{1}, x_{2}\right)=\max _{j \in J}\left|f_{j}\left(x_{1}, x_{2}\right)\right|, \quad x_{3}=1.5,
$$

where the pattern is sampled at the angles

$$
\theta_{j}=16.5+j \cdot 0.5, \quad j=1, \cdots, 147 .
$$

This means that the radiation pattern is sampled with intervals of $0.5^{\circ}$ and that the total length of the array is a fixed constant. From [10] the zero tolerance solution is known to be $\mathrm{x}^{*}=(0.432,0.926)^{T}, F^{*}=0.153 \sim-16.3 \mathrm{~dB}$ (see Fig. 5). 
Now the VTP is solved under the initial conditions $\mathrm{x}_{0}=$ $(0.5,1.0)^{T}, \delta=\left(10^{-3}, 10^{-3}\right)^{T}, \eta=1, c=0.17783 \sim-15 \mathrm{~dB}$, $\Lambda_{0}=0.05$, and $\epsilon=10^{-6}$. After 19 iterations the solution $x^{*}{ }_{\eta^{*}}=(0.441,0.931)^{T}, \eta^{*}=13.0$ is found and indicated in Fig. 5. In other words, if we realize a design where the positions of the elements are $(0.441 \pm 0.013,0.931 \pm 0.013)^{\mathrm{T}}$, then we know for sure that nowhere in the radiation pattern are the sidelobes higher than $-15 \mathrm{~dB}$.

Next, $N$ is equal to 15 . In this case we have the function

$$
F\left(x_{1}, \cdots, x_{6}\right)=\max _{j \in J}\left|f_{j}\left(x_{1}, \cdots, x_{6}\right)\right|, \quad x_{7}=3.5
$$

where the pattern is sampled at

$$
\theta_{j}=8.5+j \cdot 0.5, \quad j=1, \cdots, 163
$$

Again the solution to this ZTP is known from [10], $x^{*}=$ $(0.374,0.785,1.167,1.637,2.117,2.756)^{T}, F^{*}=0.075 \sim$ $-22.5 \mathrm{~dB}$.

We now solve the VTP under the following conditions: $\mathbf{x}_{0}=(0.5,1.0,1.5,2.0,2.5,3.0)^{T}, \delta=\left(10^{-3}, 10^{-3}, 10^{-3}\right.$, $\left.10^{-3} \cdot 10^{-3}, 10^{-3}\right)^{T}, c=0.1 \sim-20 \mathrm{~dB}, \epsilon=10^{-4}$, and $\Lambda_{0}=0.05$. The solution is reached to the required accuracy in 23 iterations and the tolerances have been increased from $10^{-3}$ to $\eta^{*} \delta_{i}=0.012$. The optimal element positions are now $(0.370,0.775,1.154,1.632,2.124,2.781)^{T}$ and the worst cases are equal to $0.1 \sim-20 \mathrm{~dB}$ within four significant digits.

\section{DISCUSSION AND CONCLUSION}

By minimizing the worst-case sidelobes in linear broadside arrays by varying both the excitation and the element positions, the usefulness of the algorithms to solve antenna design problems involving tolerances has been demonstrated. Since the analytical partial derivatives, which are required with the present version of the algorithms, are difficult to obtain when handling more complicated antenna systems, we consider the alternative possibility of applying numerical approximations to the derivatives.

A further development of the method would be to allow for structures which are symmetrical by nominal values but with nonsymmetrical deviations. This would give a more realistic treatment of the examples in this communication.

The largest worst-case optimization example considered had six variables and 171 functions and required a total CPU time of $9 \mathrm{~s}$. Of this time, approximately one-third was spent computing the functions $f_{3}$ and the partial derivatives. Clearly, by exploiting an a priori knowledge of the critical parts of the radiation patterns, the number of sample points could be considerably reduced, thereby also reducing the required computer time and storage.

\section{ACKNOWLEDGMENT}

The author wishes to thank Professor Kaj Madsen of the Institute for Numerical Analysis, Technical University of Denmark, for many helpful discussions and suggestions during the course of this work.

\section{REFERENCES}

[1] E. M. Butler, "Realistic design using large change sensitivities and performance contours," IEEE Trans. Circuit Theory, vol. CT-18. pp. 58-66, Jan. 197I.
[2] J. F. Pinel and K. A. Roberts, "Tolerance assignment in linear networks using nonlinear programming," IEEE Trans. Circuit Theory, vol. CT19. pp. $475-479$. Sept. 1972.

[3] J. W. Bandler. P. C. Liu, and J. H. K. Chen. "Worst case network tolerance optimization." IEEE Trans. Microwave Theory Tech., vol. MTT-23, pp. 630-641, Aug. 1975.

[4] J. W. Bandler. P. C. Liu. and H. Tromp. "A nonlinear programming approach to optimal design centering. tolerancing. and tuning." IEEE Trans. Circuits Syst., vol. CAS-23. pp. 155-165. Mar. 1976.

[5] J. W. Bandler and H. L. Abdel-Malek. "Modeling and approximation for statistical evaluation and optimization of microwave designs," in Proc. 7th European Microwave Conf., Copenhagen, pp. 153-157. 1977.

[6] J. W. Bandler. "Nonlinear optimization of engineering design with emphasis on centering. tolerancing and tuning. " in Large Engineering Systems, A. Wexler. Ed. New York: Pergamon. 1977.

[7] S. W. Director and G. D. Hachtel, "The simplicial approximation to design centering," IEEE Trans. Circuits Syst., vol. CAS-24, pp. 363372. July 1977.

[8] H. Schjær-Jacobsen and K. Madsen, "Algorithms for worst case tolerance optimization." to appear.

[9] K. Madsen and H. Schjar-Jacobsen. "FORTRAN program package for worst case tolerance optimization," Inst. for Numerical Analysis. Technic. Univ. Denmark, Lynghy. Rep. No. NI-78-09, July 1978.

[10] H. Schjær-Jacobsen and K. Madsen, "Synthesis of nonuniformly spaced arrays using a general nonlinear minimax optimization method." IEEE Trans. Antennas Propagat., vol. AP-24, pp. 501-506, July 1976.

[11] K. Madsen. H. Schjar-Jacobsen, and J. Voldby, "Automated minimax design of networks," IEEE Trans. Circuits Syst., vol. CAS-22, pp. 791-796. Oct. 1975 .

[12] H. Bach and J. E. Hansen. "Uniformly spaced arrays," in Antenna Theory, R. E. Collin and F. J. Zucker, Eds. New York:McGraw-Hill. 1969. ch. 5

113] R. J. Stegen, "Excitation coefficients and beamwidths of Tschebycheff arrays." Proc. IRE, vol. 41, pp. 1671-1674. Nov. 1953.

\section{Multiple Beam Feed Networks Using an Even Number of Beam Ports}

CHARLES F. WINTER

\begin{abstract}
An aperture illumination compatible with the use of an even number of adjacent beam ports in a multiple beam feed network is discussed. The antenna pattern characteristics of near-in sidelobe levels, half-power beamwidth, aperture efficiency, and feed network loss are evaluated. Maximization of the available antenna gain at adjacent beam crossover points is shown to be possible for either sequential or simultaneous operation of a receiving system. The results presented indicate that lossy feed networks are quite suitable for certain array antenna applications.
\end{abstract}

\section{INTRODUCTION}

A previous communication [1] presented results indicating that several antenna pattern characteristics should be closely evaluated in the design of receiving antenna systems using multiple beam feed networks which operate on adjacent beams either sequentially or simultaneously. The aperture illumination family considered therein was of the Taylor $(\alpha=0)$ distribution type [2] with the usual $\bar{n}$-restriction removed. It

Manuscript received January 19, 1979; revised July 10, 1979.

The author is with the Microwave/Antenna Department, Raytheon Company, Boston Post Road, Wayland, MA 01778. 